\title{
TAP Block in Postoperative Analgesia, A First Time Clinical Trial In Bangladesh
}

\author{
Bidhan Paul ${ }^{1}$, Debashis Banik ${ }^{2}$, AKM Shamsul Alam ${ }^{3}$ \\ ${ }^{1}$ Junior Consultant, Zilla Sadar Hospital, Cox'sbazar, ${ }^{2}$ Associate Professor, Department of Anesthesia, Analgesia and Intensive \\ Care Medicine, BSMMU, Dhaka, ${ }^{3}$ Professor, Department of Anesthesia, Chittagong Medical College and Hospital, Chittagong. \\ Corresponding author: E-mail: Dr. Bidhan Paul, Junior Consultant, Zilla Sadar Hospital, Cox'sbazar, Chittagong
}

\begin{abstract}
Background: In perioperative care, a reliable pain management is a vital appeal. Over recent years, Transversus Abdominis Plane (TAP) block is introduced as an important component of multimodal analgesia.
\end{abstract}

Objective: To evaluate efficacy of TAP block in postoperative analgesia for Total Abdominal Hysterectomy (TAH) with subarachnoid block (SAB) in comparison of morphine consumption and VAS score.

Methods: 60 patients were randomly allocated into 2 groups (TAP group-A \& control group-B). Standard $S A B$ was applied to all patients for elective TAH. Immediate after operation classical TAP block was performed through both Lumber Triangle Of Petit (LTOP) of group A patients. Both groups were placed in Post Anesthesia Care Unit (PACU), arranged a common standard postoperative analgesic regimen for all, observed periodically and documented it accordingly in pre-designed data sheet.

Results: TAP block prolonged the mean time of $1^{\text {st }}$ required I/V morphine (TAP vs control, mean $\pm S D$ $271.23 \pm 40.34$ vs $195.33 \pm 22.16 \mathrm{~min}$., $\left.p=0.001^{\mathrm{HS}}\right)$. Morphine requirement was also reduced (17.4 $\pm 5.4 \mathrm{vs}$ $\left.26.2 \pm 4.4 \mathrm{mg}, p=0.001^{\mathrm{HS}}\right)$. Pain VAS scores at rest and movement were also reduced at all time period ( $p \leq 0.01$ to 0.001). There was no complication attributed to the TAP block.

Conclusion: TAP block provided considerably effective postoperative analgesia in first 24 hours after major abdominal surgery like TAH.

Key words: Postoperative analgesia, TAP block, LTOP, $0.25 \%$ levobupicaine, VAS score.

(JBSA 2014; 27(1): 3-11)

\section{Introduction}

Gain and pain are more obvious in surgical procedure. Here patients may gain remedy, but pain, they pay. This pain is induced by surgical act. So, it is physician's duty to rescue the patients from surgical pain by the most possible mean. Now postoperative pain control is generally best managed by anesthesiologists, because they offer regional anesthetic techniques as well as pharmacological expertise in analgesics.

Background: Postoperative analgesia is essential to provide subjective comfort and restoration of functions like breath, cough, movement and communication effectively. From the ancient period, it was tried to do in many ways. As practiced, opioids such as morphine remain the mainstay of such regimen. However, the use of opioid only, can result significant adverse effects like nauseavomiting, sedation, respiratory depression, constipation, etc. Only NSAID use may cause GIT upset, bronchospasm, renal impairment etc. Epidural analgesia is in use, but it demands expertise; and failure rate is significant. Other techniques like rectus abdominis sheath block, paravetebral block, ilioinguinal/ iliohypogastric block, local anesthetic infiltration etc are also tested. Yet, these have flaws as they are not easy to perform, do not give adequate analgesia, do not 
produce long enough analgesic duration etc. ${ }^{1}$ The latest trend is the practice of two or more analgesic approach simultaneously called multimodal analgesia. It can produce better pain control, but, reduce the individual dose of the agent and thereby low cost, low side effect and more therapeutic safety. Over recent years, Transversus Abdominis Plane (TAP) block is introduced as an important component of multimodal analgesia. TAP is a neurofascial plane between the Internal Oblique (IO) and Transversus Abdominis (TA) Muscle of the abdominal wall. ${ }^{2}$ The abdominal wall sensory afferents course through the TAP. So, it is a novel approach to block these sensory nerves by injecting local anesthetic within the TAP, termed as TAP block. ${ }^{2}$ It is the landmark technique of block through Lumber Triangle of Petit (LTOP) has been followed in this study, also called classical blind TAP block. The block has been given by the investigator himself using inj. $0.25 \%$ levobupicaine in bilateral TAP after completion of TAH in gynae O.T. Inj. morphine and ketorolac I/V has also been used as postoperative analgesics. There was a similar study ${ }^{2}$ in Ireland, blocked by three investigators using $0.75 \%$ ropivacaine before starting TAH when patients were under general anesthesia. Their postoperative analgesia was scheduled for 48 hours with inj. Morphine via PCA, rectal acetaminophen and rectal diclofenac.

Rationale of the study: This study is devoid of general anesthesia induced hazards and residual effects. As there is residual effect of SAB, patients have not felt any pain during TAP block; rather they cooperated in identifying the site of block (LTOP). The block after operation has not bothered the surgeon to start operation. Here, a particular surgery, TAH has been chosen, as a lower abdominal major surgery gives opportunity to bilateral TAP block and maintained an equal surgical stress of the study sample. The anesthetic levobupicaine is more cardiac friendly than bupivacaine. Though ropivacaine is less cardiotoxic than bupivacaine, but not available in our country. Several randomized controlled studies have confirmed that single-shot TAP block provides analgesia up to $48 \mathrm{hrs}$, decreases postoperative morphine consumption by $70-85 \%{ }^{1}$ and minimizes its adverse effects and thereby improves compliance with post operative care including communication, mobilization, breathing exercise, early feeding etc. ${ }^{2}$
So, classical blind TAP block (study procedure) is easy to perform, technically simple, pharmacologically safe and economically cheap.

The present study was designed to evaluate the efficacy of bilateral TAP block, as part of multimodal analgesic regimen, in improved analgesia and decreased opioid consumption during $1^{\text {st }} 24$ hours after TAH under subarachnoid block (SAB) when compared with a conventional standard treatment.

\section{Literature Review:}

For scientific and clinical purposes, pain is defined by the International Association for the Study of Pain (IASP) as, "an unpleasant sensory and emotional experience associated with actual or potential tissue damage, or described in terms of such damage." This is to be distinguished from the term nociception which the IASP defines as "the unconscious activity induced by a harmful stimulus applied to sense receptors." ${ }^{3}$

Acute pain: Elicited by injury of the body tissue and activation of nociceptive transducers at the site of local tissue damage. The local injury alters the response characteristics of nociceptors and perhaps their central connection and the autonomic nervous system in the region. In general, the state of acute pain lasts for a relatively limited time and generally remits when the underlying pathology resolves. ${ }^{4}$ They are of 2 types- somatic (superficial and deep) and visceral (true visceral and parietal; localized and referred). Visceral pain is frequently associated with abnormal sympathetic or parasympathetic activity causing nausea, vomiting, sweating, changes in blood pressure and heart rate. ${ }^{\mathbf{5}}$ Postoperative pain is one of the most common forms of acute pain.

Systemic Responses to Pain: Acute pain is typically associated with a neuro- endocrine stress response that is proportional to pain intensity. Pain following abdominal and thoracic operations or trauma additionally has direct effects on respiratory function. Immobilization or bed rest due to pain in peripheral sites can also indirectly affect respiratory as well as hematological function. Moderate to severe acute pain, regardless of site, can affect nearly every organ function and may adversely influence postoperative morbidity and mortality. The latter suggests that effective 
management of postoperative pain is not only humane but is a very important aspect of postoperative care. ${ }^{5}$

\section{Postoperative Pain management:} Postoperative analgesic modalities include oral or parenteral analgesics, peripheral nerve blocks, neuraxial blocks with local anesthetics, intraspinal opioids, as well as adjunctive techniques such as TENS and physical therapy. ${ }^{5}$ These different modalities act on different sites to block pain: peripherally, on somatic and sympathetic nerves, at spinal cord level, and centrally. Combination of two or more modalities is the concept of multimodal analgesia, a balanced analgesia, an analogue of modern balanced anesthesia. ${ }^{6}$

TAP Block: The landmark technique of TAP block through LTOP was first described in 2001by Rafi as the 'one-pop technique' and was modified by McDonnell in 2007 who described a 'two pop' technique. ${ }^{1}$

The Lumber Triangle of Petit (LTOP) is formed posteriorly by the lateral border of the Latissimus Dorsi (LD) muscle and anteriorly by the posterior free border of the External Oblique (EO), with the iliac crest as the base (figure 1,2). The iliac crest serves as a fixed and easily palpable landmark.

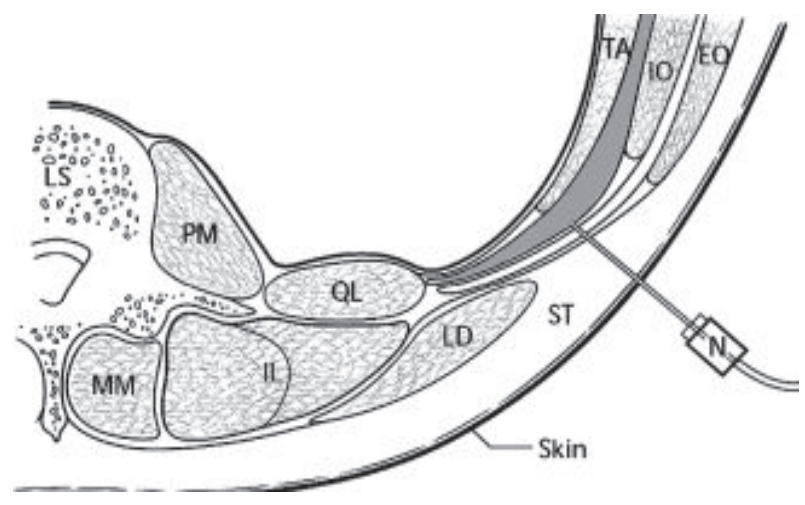

LS - lumbar spine; LD - latissimus dorsi; PM- psoas major; QL - quadratus lumborum; $\mathbf{M M}$ - multifidus muscle; IL - longissimus iliocostalis; TA- transversus abdominis; IO- internal oblique; EO - external oblique; ST - subcutaneous tissue.

Fig 1 Line drawing of a transverse section through the abdominal wall at the level of the lumbar triangle of Petit(LTOP). The needle is inserted through the triangle, is shown in the transversus abdominis plane, and the fascial layers have separated as a result of the injection of local anesthetic. ${ }^{2}$

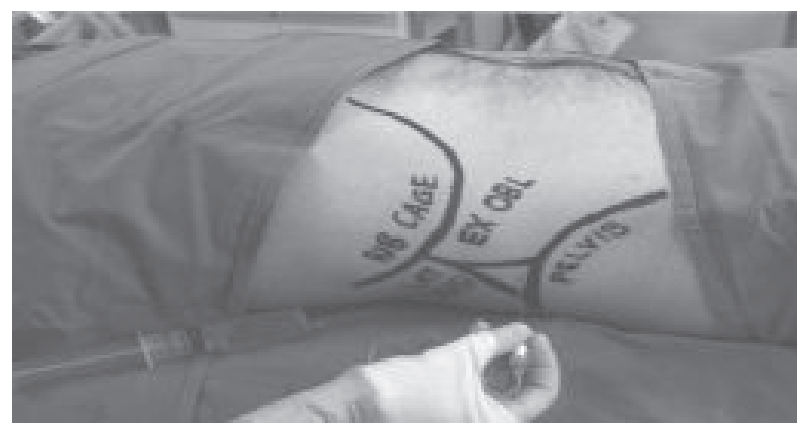

Fig.-2: Landmark insertion of TAP block through LTOP $^{7}$

Innervations: The sensory supply of the skin, muscles and parietal peritoneum of the anterior abdominal wall is derived from the anterior rami of the lower six thoracic nerves and the first lumber nerve. The intercostals $\left(\mathrm{T}_{7-11}\right)$, sub costal $\left(\mathrm{T}_{12}\right)$, iliohypogastric and ilioinguinal nerves $\left(\mathrm{L}_{1}\right)$ course through the lateral abdominal wall within the TAP before they pierce the musculature to innervate the anterior abdomen. There is extensive branching of and communication between nerves within the TAP. ${ }^{1} \mathrm{~T}_{7}$ innervates at the epigastrium, $\mathrm{T}_{10}$ at umbilicus and $\mathrm{L}_{1}$ at the groin. The LTOP and thereby the TAP is used to approach and block these neural afferents of the abdominal wall.

Landmark technique of TAP block: Requesting the patient to lift his head and shoulders from the supine position will contract the abdominal muscles and can assist palpation of the LTOP (Figure 2). The puncture site is just above the iliac crest and just posterior to the mid- axillary line within the triangle. A $22 \mathrm{G}$ or $24 \mathrm{G}$ blunt tipped $50 \mathrm{~mm}$ needle is inserted perpendicular to the skin. ${ }^{7}$ After dermal penetration, initial resistance indicates arrival of the needle tip at the EO fascia, followed by the 'two pop' sensations, one as the needle penetrates the EO fascia layer and another as it penetrates the IO fascia layer and enters the TAP (Figure 1). In recent studies the reported success rate with the landmark technique is $85 \%$ amongst experienced practitioners. ${ }^{1}$ There has been some controversy about seeking one or two 'pops' during the landmark technique of TAP block. Use of a 'two pop' technique is generally advocated and is supported by the cadaveric and imaging studies published to date. ${ }^{8}$

Other techniques: Since its first description, several modifications have been introduced, 
including the ultrasound-guided option. The use of ultrasonography is associated with substantially increased costs and requires trainings, particularly in ultrasonographic anatomy of the anaesthetized region. ${ }^{9}$ Hebbard has described a slightly different technique, called the 'oblique subcostal' TAP block, which is a combination of rectus abdominis and TAP blocks under USG guide. There are several case reports in the literature where an epidural catheter was used for continuous TAP block. It is strongly recommended that catheters should be placed only under ultrasound guidance. ${ }^{1}$

Comparison with epidural analgesia: There is no randomized controlled trial comparing TAP block and epidural analgesia. At present, TAP block is recommended in patients undergoing abdominal surgery when epidural blockade is contraindicated or not available. Epidural analgesia has the advantage of providing analgesia for visceral and somatic pain.TAP block can provide unilateral analgesia, a potential advantage in patients undergoing non-midline abdominal incision. Furthermore, TAP block can preserve bladder and lower limb motor function, thereby assisting early mobilization after surgery. The hemodynamic instability following the cardiovascular effects of epidural block is avoided. Importantly, TAP injection can be performed in sedated and ventilated patients with less risk of neuraxial injury. ${ }^{1}$

Advantages: One advantage of the TAP block is the absence of major vascular or neurological structures in this area. ${ }^{10}$ This block is easy to perform, technically simple, pharmacologically safe, economically cheap and conventional for both unilateral and bilateral approach. It mostly develops immediate analgesia and both degree and duration of analgesia increases by a single shot of injection. Single-shot TAP block provides analgesia for up to 48 hours and decreases postoperative morphine consumption by $70-80 \%$. It preserves bladder and lower motor function. $1,7,10$ There have been no reported complication to date with USG guided block. ${ }^{11}$

Limitations: Block failure is not uncommon in the skill development phase. ${ }^{12}$ Generally, TAP block have so far displayed a good safety profile. ${ }^{1}$ There is a report of liver trauma due to TAP block. In that case the block was performed before incision and liver was enlarged and extended to the right iliac crest. ${ }^{13}$ The landmark technique relies on the 'pop' sensation, some clinicians believe, is an imprecise sign. The identification of the landmarks is more challenging in the obese hence the risk of peritoneal perforation is probably higher. Some authors argue that peritoneal perforation with a small gauge sterile needle is not likely to be significant. ${ }^{7}$ Transient femoral nerve palsy is a potential complication because of the proximity of the TAP and the femoral nerve. ${ }^{1}$ Moreover, there is a risk of patient's injury (fall) if he/she is ambulated too early and the range of block involved the nerves supplying the buttock, lateral thigh or the region supplied by the femoral nerve. ${ }^{9}$ There is always the possibility of underreported minor complications. ${ }^{1}$ Local anesthetic toxicity could also occur due to the large volumes required to perform this block specially if it was done bilaterally. As with any regional technique, careful aspiration will help avoid intravascular injection. ${ }^{11}$ Anesthetists using TAP block should be aware of the possibility of visceral damage if the needle is advanced too far inadvertently. The catheter technique has the potential to result in more complications compared with single shot. ${ }^{1}$

\section{Materials and Methods}

This prospective, non blind, randomized, controlled trial was studied in Department of Anesthesia and Intensive Care, Chittagong Medical College Hospital and Department of Anesthesia, Analgesia and Intensive Care Medicine (AAICM), BSMMU, Dhaka from January 2010 to June 2011. Among the women undergoing routine TAH with lower transverse incision under subarachnoid block (SAB) and given informed consent for bilateral TAP block, 60 patients were collected during daily preanesthetic assessment (PAA) as first come first basis. They were of ASA I - II and BMI d" $35 \mathrm{~kg} /$ $\mathrm{m}^{2}$ and divided randomly by lottery into two equal groups A (TAP group) and B (non TAP group). Exclusion criteria were block site infection, refusal for TAP block, intolerance to opioid, H/O sensitivity to prescribed analgesic and $\mathrm{H} / \mathrm{O}$ chronic back pain with daily consumption of analgesics.

\section{Materials:}

1. Equipments- Regional block needle (here, we used 20G I.V canula trocher making blunt slightly), two disposable syringes of 10c.c, 
disinfectant for scrubbing the TAP blocking sites, sterile dressing etc.

2. Drugs- a) Inj. 0.25\% Levobupivacaine, inj. Morphine 15mg and inj. Ketorolac 30mg b) Others- inj. Prochlorperazine 12.5mg, inj. Ondensatron $8 \mathrm{mg}$, inj. Naloxone $4 \mathrm{mg}$ etc.

3. Relevant books, journals and internet searching for literatures.

\section{Methods:}

Study was conducted in full record with ethical principles.

Pre-anesthetic assessment (PAA) was done at the day before surgery. Sample was selected accordingly and briefed about the study and procedure, written informed consent were obtained including counseling about VAS score for post operative pain. The first page of the predesigned data collection sheet was filled up including particulars of the patient, diagnosis etc. This page was separated from the others by putting a serial no. and made anonymity of the real data page.

At the day of surgery, a patient was received into operation theatre. Once again, she was reassured. The baseline parameters were measured and documented in data sheet, an I.V channel was opened and preload was done with the Hartman's solution of about $500 \mathrm{ml}$. A standard SAB was applied to the patient with $15 \mathrm{mg}$ hyperbaric bupivacaine and Fentanyl 25 microgram through a Quincke's 25G spinal needle at the level of L2-3 or L3-4 intervertebral space in sitting position. Intraopeative maintaince was done with due monitoring.

After completion of TAH, inj. Ketorolac 30mg I.V was given stat and 8 hourly to both groups of patient and vital signs, VAS score etc were measured. Soon after, if there was no exclusion criteria then bilateral TAP block was performed to group A as-

1. Patient in supine position without hip or pelvic flexion.

2. The ipsilateral arm was raised above the head to accentuate the latissimus dorsi. The lateral fat pad of patient might be retracted superiorly such that the iliac crest was easily palpable in almost all cases.
3. We should found the anterior superior iliac spine and advanced above the iliac crest backwards till the lateral edge of latissimus dorsi muscle was felt. The Triangle of Petit is located anterior to this muscle. After the identification of this point, aseptic skin preparation was done accordingly.

4. Then the needle was introduced with perpendicular to the skin just above the iliac crest until the characteristic " $2^{\text {nd }} P O P$ " was identified and entering the target place, i.e., Transversus Abdominis Plane (TAP).

5. After aspiration test, $20 \mathrm{ml}$ of $0.25 \%$ levobupivacaine was injected in one side with intermittent aspiration test to prevent intravascular injection (first $2 \mathrm{ml}$ to test easy flow and hypersensitivity). Now the needle was withdrawn and sterile dressing was placed.

6. Same sequence was done for opposite side block.

7. Sterile dressing pad was also placed over triangle of Petit bilaterally in group B to avoid easy biasness during post operative care.

Group-B patients were managed according to protocol procedure but devoid of TAP block. All Patients were in PACU and observed at 1,2,4,6,12 and 24 postoperative hour. All observations were recorded in pre-prepared data sheet accordingly by the investigator with the help of trained PACU nurses.

When patient's VAS score was $>3$, she was treated with inj. morphine $2 \mathrm{mg} \mathrm{I} / \mathrm{V}$ as rescue analgesic with inj prochlorperazine $12.5 \mathrm{mg} \mathrm{I} / \mathrm{M}$ during first dose. Subsequent doses of injection morphine were $1 \mathrm{mg}$ I/V for same purpose. Rescue antiemetic was offered to the patients complained of nausea and vomiting. After 24 hours dressing over the block site was checked for any sign of infection.

\section{Methods of statistical analysis:}

Relevant information was recorded in pre-designed data collection sheet and later on was compiled on master chart. The quantitative data were expressed in terms of mean (standard deviation) and comparison was done employing student's " $\mathrm{t}$ " test (unpaired). P value d" 0.05 was considered as significant. Statistical calculation was done using statistical package for social science (SPSS) version 17. 


\section{Result}

There was no significant difference between two groups in terms of their age, body weight and basal metabolic index (BMI) (Table 1). The ASA status, educational status and history of prior abdominal surgery were also identical in both groups. In all patients of group A, the triangle of Petit was located easily on palpation, the tranversus abdominis neurofascial plane was localized after one to two attempts and the block was performed without complication. The length of needle introduced for TAP block was $33-41 \mathrm{~mm}$.

In both groups the duration of TAH under SAB was of no significance of difference $(p=0.617)$ (Table 2 ), but first I/V morphine requirement mean time \pm SD was $271.23 \pm 40.34$ (range 175-355) minutes in group A and 195.33 \pm 22.16 (range 165-270) minutes in group B (Fig.3). It was of highly significance of difference ( $p=0.001)$. It was the mean time between application of SAB and use of I/V morphine loading dose when patient's VAS score for pain $>3$.

Postoperative mean consumption of $\mathrm{I} / \mathrm{V}$ morphine was lower $\left(\mathrm{p}=0.001^{\mathrm{HS}}\right)$ in group A than group $\mathrm{B}$ at all time points as $1^{\text {st }} 6 \mathrm{hrs}, 2^{\text {nd }} 6 \mathrm{hrs}$ and last $12 \mathrm{hrs}$ (Table 3). The range of total $24 \mathrm{hrs} \mathrm{I} / \mathrm{V}$ morphine requirement was $10-28 \mathrm{mg}$ in group $\mathrm{A}$ and $20-35 \mathrm{mg}$ in group B (not shown in graph). Pain VAS scores at rest were significantly lower (i.e. better controlled) in group A than group B. It was with highly significance of difference $(\mathrm{p}=0.001)$ at $1^{\text {st }}$, $2^{\text {nd }}, 4^{\text {th }}$ and $24^{\text {th }}$ hr of postoperative time points but very significant $(\mathrm{p}=0.01)$ at $6^{\text {th }}$ and $12^{\text {th }} \mathrm{hr}$ time points (Fig 4). On the contrary, VAS scores at movement were lower in same group with highly significant value of difference $(\mathrm{p}=0.001)$ at all postoperative time points(Fig.5).

Table I Patient's baseline characteristics

\begin{tabular}{lcccccc}
\hline Characteristics & Group & Maximum & Minimum & Mean & \pm SD & P value \\
\hline Age (yrs) & A & 55 & 34 & 41.77 & \pm 5.34 & $0.283^{\text {NS }}$ \\
& B & 52 & 37 & 43.00 & \pm 3.20 & \\
Weigh $(\mathrm{kg})$ & A & 65 & 49 & 56.36 & \pm 3.76 & $0.601^{\text {NS }}$ \\
& B & 65 & 47 & 55.83 & \pm 4.07 & \\
BMI $\left(\mathrm{kg} / \mathrm{m}^{2}\right)$ & A & 25.70 & 20.80 & 23.46 & \pm 1.24 & $0.657^{\mathrm{NS}}$ \\
& B & 25.40 & 20.00 & 23.31 & \pm 1.35 & \\
\hline
\end{tabular}

Group- A ( $\mathrm{n}=30)$ : Case (TAP); Group- B ( $=30)$ : Control (Non TAP)

$\mathrm{n}$ : number of TAH patient in each group BMI: Basal metabolic index

$\mathrm{P}>0.05$ - Non-Significant (NS) $\quad \mathrm{P}<0.05$ - Significant (S)

$\mathrm{P}<0.01$ - Very significant (VS) $\quad \mathrm{P}<0.001$ - Highly significant (HS)

Test used: unpaired student's 't' test of significance of difference

Table-II Duration needed for total abdominal hysterectomy

\begin{tabular}{lccc}
\hline $\begin{array}{l}\text { Characteristics } \\
\text { (Time in }\end{array}$ & $\begin{array}{c}\text { Group A } \\
(\mathrm{n}=30)\end{array}$ & $\begin{array}{c}\text { Group B } \\
(\mathrm{n}=30)\end{array}$ & $\begin{array}{c}\text { P- } \\
\text { value }\end{array}$ \\
minutes $)$ & & & \\
\hline Mean \pm SD & $97.66 \pm 12.29$ & $99.16 \pm 10.75$ & $0.617^{\mathrm{NS}}$ \\
Minimum & 65.0 & 70.0 & \\
Maximum & 125.0 & 120.0 & \\
\hline
\end{tabular}

Test used: unpaired student's ' $t$ ' test of significance of difference

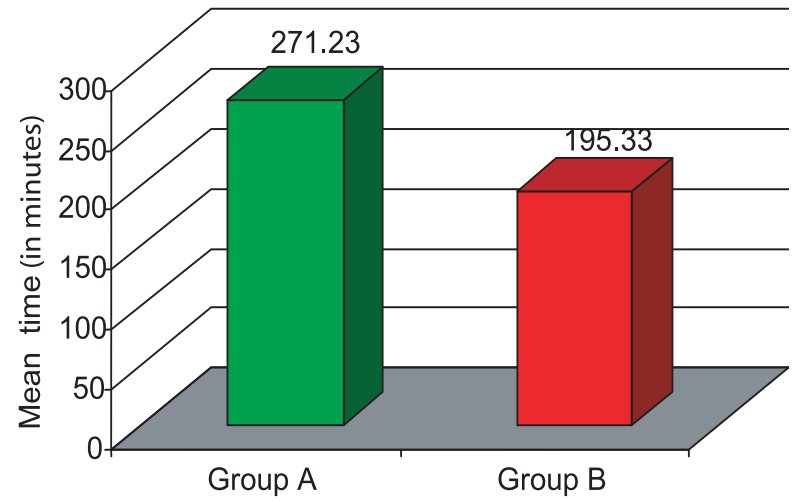

Fig 3 First morphine requirement mean time after $S A B$ 
Table-III Distribution of I/V morphine consumption ( $\mathrm{mg}$ )

\begin{tabular}{lcccccc}
\hline Parameters & Group & Maximum $(\mathrm{mg})$ & Minimum $(\mathrm{mg})$ & Mean & $\pm \mathrm{SD}$ & P value \\
\hline $1^{\text {st }} 6$ hours & $\mathrm{A}$ & 15 & 04 & 8.20 & \pm 2.50 & $0.001^{\mathrm{HS}}$ \\
& $\mathrm{B}$ & 16 & 08 & 11.0 & \pm 1.70 & \\
$2^{\text {nd }} 6$ hours & $\mathrm{A}$ & 07 & 02 & 4.40 & \pm 1.30 & $0.001^{\mathrm{HS}}$ \\
& $\mathrm{B}$ & 10 & 05 & 7.80 & \pm 1.30 & \\
Last 12 hours & $\mathrm{A}$ & 08 & 02 & 4.80 & \pm 1.60 & $0.001^{\mathrm{HS}}$ \\
& $\mathrm{B}$ & 10 & 05 & 7.40 & \pm 1.40 & \\
\hline
\end{tabular}

Morphine consumption in milligram

Values are Mean $\pm \mathrm{SD}$

Test used: unpaired student's 't' test of significance of difference

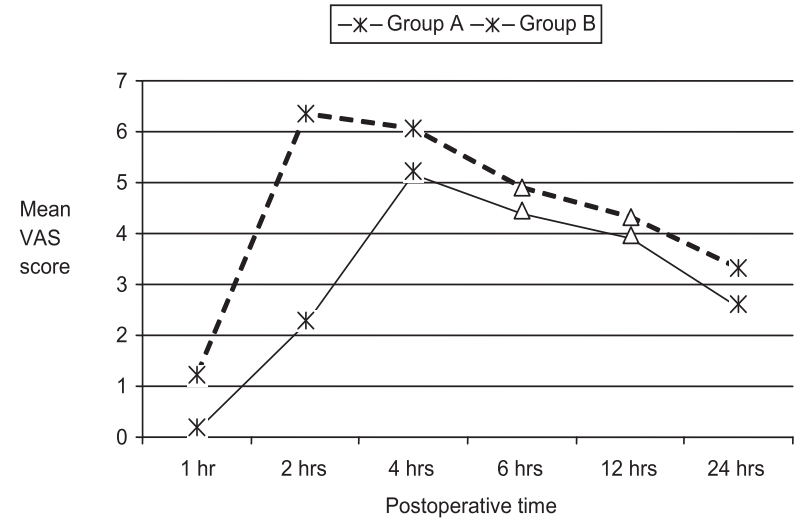

Fig 4 Distribution of VAS score at rest

\section{Discussion}

A multimodal approach to postoperative analgesia after TAH is rational because of the need to block nociceptive transmission from both the abdominal wall incision and from visceral sites.

The present study reveals that the mean time of first morphine requirement as rescue analgesic in TAP group is longer with high significance ( 273 vs $195 \mathrm{~min}, \mathrm{p}=0.001$ ). The study by John Carney et $\mathrm{al}^{2}$ also showed the same significance but the real time interval was very shorter (median $45 \mathrm{vs}$ $12.5 \mathrm{~min}, \mathrm{p}<0.001)$. Probably, because of their study was under G.A and there was no residual effect of SAB, like present study. So, there is additive analgesic effect of neuraxial anesthesia with TAP block in our study.

Mean I/V morphine consumption in postoperative 24 hours is about $33.6 \%$ reduced in TAP group of

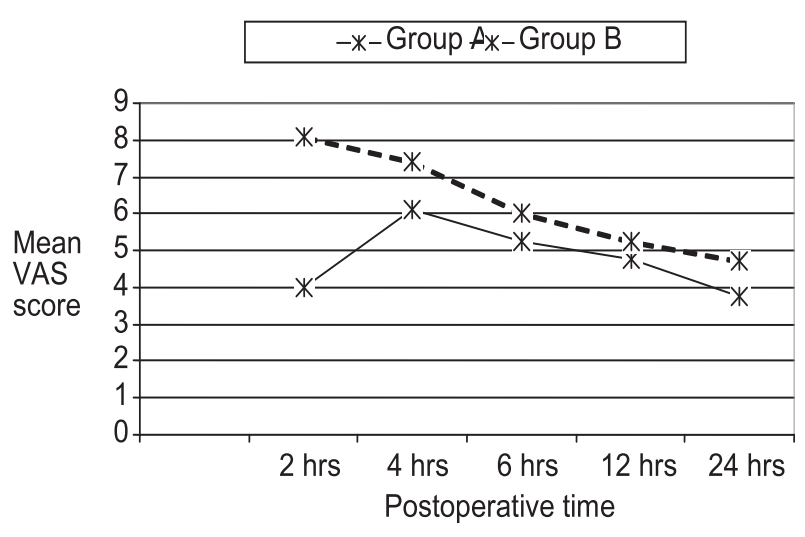

Fig 5 Distribution of VAS score at movement

patients in this study. But it was reduced by $46 \%$ in the study of John Carney et $\mathrm{al}^{2}$. Probably, patient's body weight (about $55 \mathrm{vs} 75 \mathrm{~kg}$ in the present and marked study) and the type and strength of anesthetic used $(0.25 \%$ levobupivacaine vs $0.75 \%$ ropivacaine) are the important reasons for this discrepancy. On the contrary, there are evidence of morphine reduction by $70 \%$ in the study by $\mathrm{McDonell}$ et al for bowel surgery ${ }^{14}$ under G.A and cesarean section ${ }^{\mathbf{1 5}}$ under SAB. Even John D Scharine ${ }^{\mathbf{1 2}}$ reported that both of his TAP block cases did not use any of the narcotic analgesic option available to them whereas maintained low pain scores (0-4).

In this study, postoperative VAS pain scores at rest and movement are reduced after TAP block at most but not same at all time points assessed $\left(\mathrm{p}<0.01^{\mathrm{VS}}\right.$ to $<0.001^{\mathrm{HS}}$ ). Almost the similar results were found in the study by John Carney et $\mathrm{al}^{2}$ and John G McDonnell et $\mathrm{al}^{14}$ ( $\mathrm{p}<0.05$ to $\left.<0.001\right)$. 
This study shows no documented complication. There is no respiratory depression (resp. rate d"6/ min.) in either group. Only one patient in group A needed the highest $28 \mathrm{mg}$ of morphine, an outlier, might be a case of block failure.

There is better postoperative hemodynamic stability at group A (TAP group) in terms of lower pulse rate, systolic and diastolic blood pressure (non significance to highly significance of difference) due to better pain control and less stress response than group B.

The limitation of this study are of its non blindness and small sample size, though true blinding may not possible in TAP block patients. VAS (visual analogue score) pain scores should be justified by another scale at least like VRS (verbal rating scale), NRS (numerical rating scale) etc. There are seven patients in TAP group consume morphine more than double of the minimum consumption $(10 \mathrm{mg})$ recorded in a case of the group. It is not identified whether those cases were partial block or block failure.

\section{Conclusion}

We may conclude that, TAP block offers more significant analgesia after total abdominal hysterectomy. There is no complication detected due to TAP block. Tap block is technically easy, pharmacologically safe and economically cheap. So, it seems to hold considerable prospect as part of multimodal analgesic regimen after lower abdominal surgery like TAH, LSCS, appendectomy, prostatectomy, herniotomy etc.

Further study is suggestive of intra operative TAP block in abdominal surgery before closure of peritoneum to avoid inadvertent visceral injury, unexpected intra or extra peritoneal block and thereby almost ensure that injection of local anesthetic is within the TAP. It might be the real alternative for ultrasound guided TAP block. Use of ketorolac may be reduced or replaced by paracetamol in future studies and clinical practice. Comparison study between epidural and TAP block analgesia is a demand also. There is a large scale study of TAP block require to detect plasma concentration of local anesthetic and further establish the block safety thereby.

\section{References}

1. Zorica Jankovic.Transverses Abdominis Plane Block:The Holy Grail of Anaesthesia For (lower) Abdominal Surgery. Periodicum Biologorum,[online]2009;111:203-8 http:// www.google.com/search? $q=$ transversus + abdominis + plane + block\&hl=en\&pr $m d=i v n s \&$ e $i=w c 0 R T q S 5 G I_{-} j$ r AfEtKyIBA\& start $=10 \& s a=N$ (accessed $13 \mathrm{Feb}, 2010)$

2. Carney J, McDonnell JG, Ochana A, Bhinder R, Laffey JG. The Transverses Abdominis Plane Block Provides Effective Postoperative Analgesia in Patients Undergoing Total Abdominal Hysterecromy. Anestha Analg,[online] 2008; 107( 6): 2056-60 http:// www.anesthesia-analgesia.org/content/107/ 6/2056.long (accessed 10 Jan,2010)

3. Barrett KE, Barman SM, Boitano S, Brooks HL (eds). Pain and Temperature. In: Ganong's Review of Medical Physiology, $23^{\text {rd }}$ edition. New D'elhi: Tata McGraw Hill Education (Lange), 2010: 168

4. Dureja GP. Handbook of Pain Management. New Delhi: Elsevier, 2004: 310

5. Morgan GE, Mikhail MS, Murray MJ. Pain management. In: Clinical Anaesthesiology, $4^{\text {th }}$ edition (international). New York: McGrawHill, 2006: 362-396

6. Aitkenhead AR, Rowbotham DJ, Smith G. eds. Postoperative pain. In: Textbook of Anaesthesia, $4^{\text {th }}$ edition (international). Toronto: Churchill Livingstone, 2001: 553

7. Katrina Webster.The transversus abdominis plane (TAP) block: Abdominal plane regional anaesthesia. Update in Anaesthesia [online] http://home.vicnet.net.au/ gatepain / PM006.pdf (accessed 15 Feb,2010)

8. McDonnell J, O’Donnell B, Farrell T, Gough N, Tuite D, Power C, Laffey J. Transversus abdominis plane block: A Cadaveric and Radiological Evaluation. Regional anaesthesia and pain medicine [online] 2007; 32: 399-404 http://www.researchgate.net/publication/ 5883745_Transversus_abdominis_plane_ block_a_cadaveric_and_radiological_evaluation (accessed 24 August,2010) 
9. Leszek Urbanczak. Transverses Abdominis Plane Block. Resmedica Anaesthesiology Intensive Therapy, [online] 2009; 3: 133-135 http://anestezjologia.net/en/articles /item/ 10062/transversus_abdominis_plane_block (accessed $14 \mathrm{Feb}, 2010$ )

10. L Pillay. Transverses abdominis plane block.[online]13 march 2009:1-24 http:// anaesthetics.ukzn.ac.za/Libraries / $F M M \_R \_B \_2009 / T r a n s v e r s u s \_a b d o m i n i s \_$ Plane_Block_Dr_L_Pillay.sflb.ashx(accessed 20 April,2010)

11. Karim Mukhtar. Transverses abdominis plane (TAP) block. The Journal of NYSORA [online] 2009; 12: 28-33 http://www.nysora.com/files / uploaded/JNYSORA/Volume12/(v12p2833)TAPBlock.pdfs (accessed 25 April,2010)

12. John D Scharine. Bilateral Transverses Abdominis Plane Nerve Block for Analgesia Following Cesarean Delivery: Report of 2 Cases. AANA Journal, [online] April 2009; 77( 2 ): 98-102 http://www.google.com/ search? $q=$ transversus + abdominis + plane $+b l o$
ck\&hl=en\&prmd=iuns\&ei=a9YRTrgmivitB-_$w J 4 I \& s t a r t=100 \& s a=N$ (accessed $14 \mathrm{Feb}, 2010)$

13. Farooq M, Carey M. A case of liver trauma with a blunt regional anesthesia needle while performing transversus abdominis plane block. Reg Anesth Pain Med [online] 2008; 33: 274 http://www.ncbi.nlm.nih.gov/pubmed/ 18433683 (accessed 19 August,2010)

14. McDonnell JG, O'Donnell BD, Curley G, Heffernan A, Power C, Laffey JG. Analgesic efficacy of transversus abdominis plane (TAP) block after abdominal surgery: a prospective randomized controlled trial. Anesth Analg [online] 2007;104:193-7 http://www.anesthesia -analgesia.org / content/104/1/193.full (accessed 07 Feb,2010)

15. McDonnell JG, Curley G, Carney J, Benton A, Costello J, Maharaj CH, Laffey JG. The analgesic efficacy of transversus abdominis plane block after cesarean delivery: a randomized controlled trial. Anesth Analg [online] 2008; 106:186-91 http:// www.anesthesia-analgesia.org/content/106/ 1/186.long (accessed 20 April,2010) 